\title{
Avaliação de treinamento cirúrgico na graduação de medicina
}

\section{Evaluation of surgical training in medical school}

\author{
Kátia Sheylla Malta Purim; laila Djensa Souza dos Santos²; Gabriel Treml Murara3; Eliane Mara Cesário Pereira Maluf ${ }^{4}$; \\ Julio Wilson Fernandes ${ }^{5}$; James SkInOVSKy, TCBC-PR ${ }^{6}$
}

\section{R E S U M O}

\begin{abstract}
Objetivo: Avaliar a efetividade de um treinamento de técnicas cirúrgicas em pata de porco durante a graduação em medicina. Métodos: Estudo transversal com 87 acadêmicos de medicina voluntários, provenientes de diferentes períodos da graduação, que participaram de uma oficina básica para realização de incisões, suturas, biópsias, retalhos, enxertos, sob supervisão direta. Foram utilizados questionários autoaplicáveis pré e pós-treinamento. Resultados: O ensino de suturas, biópsias e correção de orelha mostrou-se efetivo para o aprendizado de habilidades mínimas exigidas para as técnicas propostas $(p<0,005)$, bem como, das excisões e retalhos exercitados, à exceção do enxerto $(p>0,97)$. Conclusão: A abordagem metodológica e o monitoramento de reprodução das técnicas mostraram-se adequados. O treinamento oferecido complementou as habilidades cirúrgicas cutâneas dos graduandos de medicina.
\end{abstract}

Descritores: Medicina. Ensino. Educação médica. Educação de graduação em medicina. Procedimentos cirúrgicos ambulatoriais.

\section{INTRODUÇÃO}

$\mathrm{O}$ processo de ensino na medicina, principalmente nas áreas cirúrgicas, ocorre pela observação do ato cirúrgico e pela participação direta do estudante, realizado, entre outros métodos, através de tentativa e erro ${ }^{1,2}$. O interesse demonstrado pelo aluno pode abrir oportunidades de aprendizado mais aprofundado, quando acompanha cirurgias e realiza atividades extracurriculares, aliado aos ensinamentos tradicionais ministrados pelos cursos de Medicina. A qualidade destas experiências no campo da cirurgia pode influenciar a aquisição de habilidades técnicas 3,4.

Desta forma, é fundamental a avaliação objetiva das técnicas operatórias visando detectar deficiências que possam ser corrigidas e preparar o acadêmico para a realização adequada dos procedimentos cirúrgicos básicos requeridos na vida profissional ${ }^{5,6}$. Muitas estratégias pedagógicas têm sido desenvolvidas para suprir as necessidades do ensino da técnica operatória durante a graduação ${ }^{1-}$ ${ }^{3}$. O modelo com patas de porco é um dos mais utilizado nos programas de residência médica em dermatologia nos EUA para ensino prático das operações sobre a pele e ane$\mathrm{xOS}^{2}$. Eticamente aceito e reprodutível, este modelo simula a pele humana com custo reduzido, e o estudante pode ser avaliado quanto as suas habilidades ao final do treinamento'.
Diante do exposto, elaborou-se um estudo com o objetivo de avaliar o treinamento cirúrgico em patas de porco em um grupo heterogêneo de estudantes de Medicina.

\section{MÉTODOS}

O enfoque metodológico adotado foi transversal com abordagem quantitativa. O trabalho de campo se constituiu de uma oficina de cirurgia cutânea montada como projeto de extensão e desenvolvida em patas de porcos refrigeradas. O projeto foi submetido e aprovado pelo Comitê de Ética da instituição (protocolo CEP -UP 165/ 2009).

O público-alvo foi composto por estudantes maiores de 18 anos, regularmente matriculados no Curso de Medicina, voluntários para este estudo. Ocorreram duas oficinas cada uma delas formada com metade do grupo, com divisão por afinidade, para melhor locação dos participantes e condições didáticas.

Como parte das estratégias de avaliação, utilizou-se de um instrumento de coleta de dados elaborado em forma de questionário autoaplicável, respondido antes e depois do treinamento, com roteiro estruturado composto por perguntas fechadas, para atender os principais pon-

Trabalho realizado no Curso de Medicina da Universidade Positivo.

1. Professora das Disciplinas de Dermatologia e Cirurgia Ambulatorial do Curso de Medicina da Universidade Positivo- Curitiba - PR-BR; 2. Acadêmica $5^{\circ}$ ano do Curso de Medicina da Universidade Positivo; 3. Acadêmico $6^{\circ}$ ano do Curso de Medicina da Universidade Positivo; 4. Professora da Disciplina de Saúde da Família do Curso de Medicina da Universidade Positivo- Curitiba - PR-BR; 5. Professor das Disciplinas de Cirurgia Ambulatorial e Clínica Cirúrgica; 6. Professor das Disciplinas de Cirurgia Ambulatorial e Clínica Cirúrgica. 
tos de interesse do treinamento. Os métodos utilizados em cada oficina foram os mesmos: questionário, as mesmas técnicas, professores, monitores, disponibilidade de material e roteiro de exercícios.

Os alunos foram previamente orientados quanto ao aspecto operacional da oficina e preenchimento dos questionários, recebendo kits cirúrgicos individualizados contendo tesoura, pinça anatômica, pinça dente de rato, porta agulhas, cabo e lâminas de bisturi, fios agulhados, luvas de procedimento e punch (instrumento cirúrgico tipo saca-bocado constituído por cabo e borda circular cortante). O roteiro dos exercícios práticos incluía biópsias, incisões, excisões, movimentos dos tecidos, retalhos, noções de enxertos, suturas e técnica de correção das chamadas "dog ears" nas extremidades das excisões com inadequada relação largura/comprimento. No centro cirúrgico experimental os estudantes foram distribuídos em dez mesas cirúrgicas, formando pequenos grupos para realização das atividades. Cada exercício foi precedido de explicação detalhada, bem como demonstração em multimídia.

Todos os alunos foram diretamente supervisionados na execução de cada técnica e estimulados a esclarecer dúvidas com os monitores e professores. A proporção de professores foi de um para cada 12 alunos e de monitores foi de um para cada grupo de quatro ou cinco estudantes que constituíram uma equipe fixa por mesa cirúrgica. Todos os instrutores conheciam o conteúdo e a metodologia proposta, possuindo domínio sobre o programa de exercícios cirúrgicos aplicados. Ao final do treinamento ocorreu nova avaliação.

Os dados obtidos foram codificados e as variáveis categóricas, apresentadas em percentuais foram analisadas pelo teste do qui quadrado. Considerou-se como nível de significância estatística $p<0,05$.

\section{RESULTADOS}

Dos 87 estudantes de medicina que participaram do treinamento, $48(55,2 \%)$ eram do sexo masculino e $39(44,8 \%)$ eram do sexo feminino. Destes acadêmicos, $53(60,5 \%)$ apresentavam idade entre 20 e 23 anos, 66 $(75,8 \%)$ estavam entre o $6^{\circ}$ e o $9^{\circ}$ período do curso de medicina e 70 (80,5\%) tinham interesse em área cirúrgica. As características dos participantes são mostradas na tabela 1

O treinamento das suturas propostas, exceto o ponto de Donati contínuo e sutura intradérmica, foi efetivo para o aprendizado das técnicas ensinadas $(p<0,005)$, bem como a biópsia por punch (Figura 1), e a técnica de correção das chamadas dog ears.

As excisões e retalhos também demonstraram uma significativa apropriação da técnica básica apesar das variações no gestual cirúrgico (Figura 2).

O ensino de enxertos não foi estatisticamente significante para o aprendizado da técnica $(p>0,97)$. Na tabela 2 são exibidos os resultados referentes ao aprendizado deste treinamento.

\section{DISCUSSÃO}

Segundo as Diretrizes Curriculares Nacionais do Curso de Graduação em Medicina do Ministério da Educação (MEC), o estudante deve saber realizar procedimentos clínicos e cirúrgicos necessários para o atendimento ambulatorial e de urgências e emergências. O curso de graduação precisa utilizar metodologias que privilegiem a coparticipação do aluno no processo educacional, inserindo-o precocemente em atividades práticas relevantes para a vida médica ${ }^{7,8}$. Segundo estes preceitos, o modelo experimental de cirurgia cutânea em patas de porco pode ser

Tabela 1 - Características dos participantes do treinamento cirúrgico em pata de porco

\begin{tabular}{llrr}
\hline Características da amostra & & $\mathrm{n}$ & $\%$ \\
\hline Sexo & Feminino & 39 & 44,8 \\
& Masculino & 48 & 55,2 \\
Idade ao realizar o curso & Não respondeu & 4 & 4,6 \\
& 19 anos & 5 & 5,7 \\
& 20 aos 23 anos & 53 & 60,5 \\
& 24 aos 27 anos & 20 & 22,9 \\
Período do curso de medicina & $>28$ anos & 5 & 5,6 \\
& $3^{\circ}$ ao $5^{\circ}$ & 18 & 20,6 \\
Interesse em área cirúrgica & $6^{\circ}$ ao 9o & 66 & 75,8 \\
& $10^{\circ}$ ao $12^{\circ}$ & 3 & 3,4 \\
& Não respondeu & 4 & 4,6 \\
& Sim & 70 & 80,5 \\
\hline
\end{tabular}




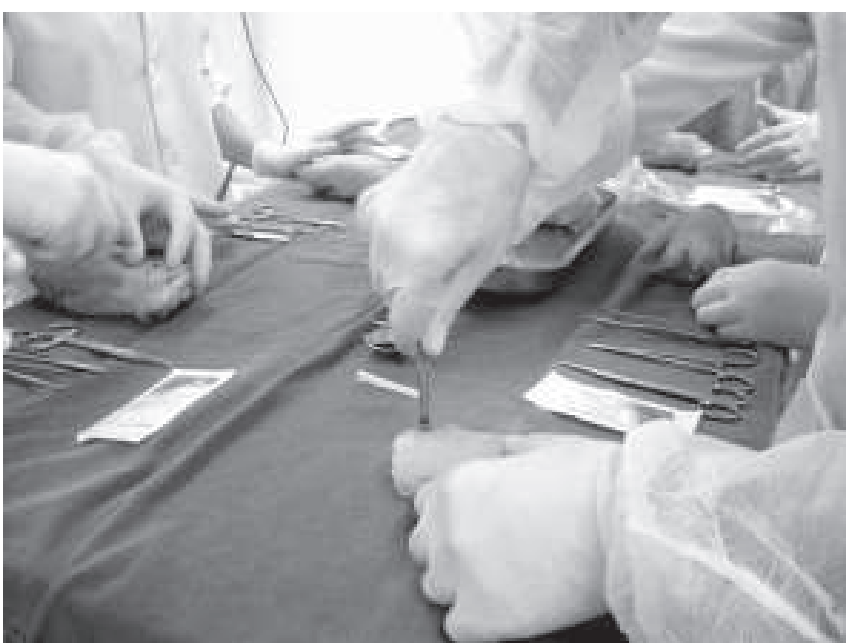

Figura 1 - Grupo de alunos treinando biópsia por punch.

utilizado pelas instituições de ensino, pois permite a avaliação do aluno de forma mais objetiva e o ajuda nas bases e aquisição de habilidades ${ }^{3}$.

Neste trabalho, a metodologia teve uma ótima receptividade por parte dos alunos e docentes por fornecer uma boa noção dos procedimentos, e não contrariar as leis vigentes que proíbem a utilização de animais para experimentos ou apresente riscos para os participantes. O planejamento didático manteve o desafio com a apropriação e reconstrução do conhecimento de forma significativa e consistente, e o compromisso com sua avaliação.

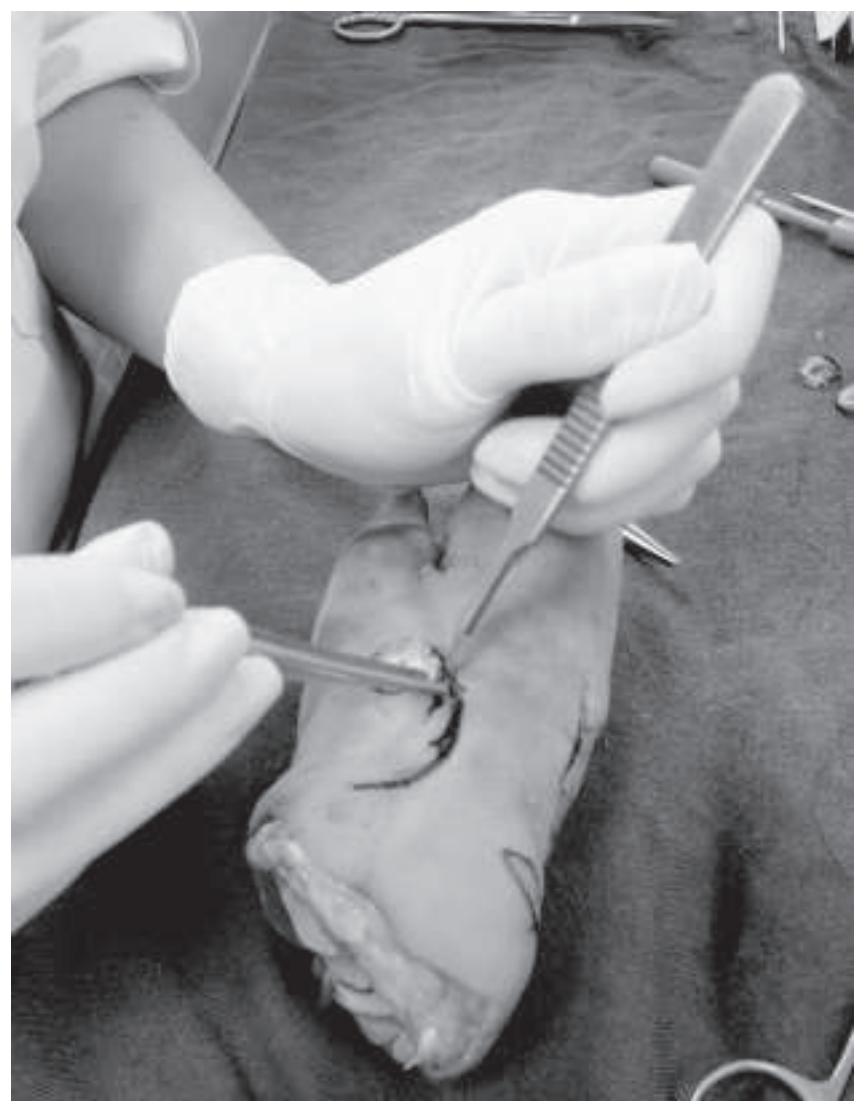

Figura 2 - Aluna canhota usando bisturi para realizar retalho em pata de porco durante o treinamento cirúrgico.

Tabela 2 - Aprendizado das técnicas básicas ensinadas durante a oficina de cirurgia experimental.

\begin{tabular}{|c|c|c|c|c|c|c|c|c|c|}
\hline \multirow[t]{2}{*}{ Técnica cirúrgica } & \multicolumn{4}{|c|}{ Habilidade prévia ao treinamento } & \multicolumn{4}{|c|}{$\begin{array}{cc}\text { Habilidade } & \text { pós-treinamento } \\
\text { Sim } & \text { Não }\end{array}$} & \multirow[t]{2}{*}{$P$} \\
\hline & $\mathrm{n}$ & $\%$ & $\mathrm{n}$ & $\%$ & $\mathrm{n}$ & $\%$ & $\mathrm{n}$ & $\%$ & \\
\hline \multicolumn{10}{|l|}{ Suturas } \\
\hline Suturas geral & 72 & 82,8 & 15 & 17,2 & 87 & 100 & 0 & 0 & $<0,001$ \\
\hline Simples & 72 & 82,5 & 15 & 17,2 & 86 & 98,9 & 1 & 1,1 & $<0,001$ \\
\hline Donati contínuo & 15 & 17,2 & 72 & 82,8 & 16 & 18,4 & 71 & 81,6 & 0,84 \\
\hline Intradérmica & 48 & 55,2 & 39 & 44,8 & 56 & 64,4 & 31 & 35,6 & 0,22 \\
\hline Geral & 23 & 26,5 & 64 & 73,5 & 86 & 98,9 & 1 & 1,4 & $<0,001$ \\
\hline Avanço & 10 & 11,5 & 77 & 88,5 & 83 & 95,4 & 4 & 4,6 & $<0,001$ \\
\hline$V-Y$ & 18 & 20,7 & 69 & 79,3 & 75 & 86,2 & 12 & 13,8 & $<0,001$ \\
\hline Rotação & 7 & 8,0 & 80 & 92,0 & 82 & 94,3 & 5 & 5,7 & $<0,001$ \\
\hline Transposição & 4 & 4,6 & 83 & 95,4 & 79 & 90,8 & 8 & 9,2 & $<0,001$ \\
\hline À distância & 1 & 1,1 & 86 & 98,9 & 12 & 13,8 & 75 & 86,2 & 0,004 \\
\hline Excisão & & & & & & & & & \\
\hline $\begin{array}{l}\text { Geral } \\
\text { Enxertot }\end{array}$ & 25 & 28,7 & 62 & 71,3 & 78 & 88,5 & 9 & 11,5 & $<0,001$ \\
\hline $\begin{array}{l}\text { Geral } \\
\text { "DOG EARS" } \neq\end{array}$ & 2 & 2,5 & 79 & 97,5 & 4 & 5,0 & 77 & 95,0 & 0,97 \\
\hline Geral & 14 & 16,3 & 72 & 83,7 & 80 & 93,2 & 6 & 6,8 & $<0,001$ \\
\hline
\end{tabular}

+ Foram excluídos os indivíduos que não responderam o pós-teste (seis perdas).

F Foram excluídos os indivíduos que não responderam o pós-teste (uma perda). 
A reprodução dos exercícios cirúrgicos durante o treinamento foi acompanhada passo a passo permitindo que a maioria das técnicas operatórias ministradas tivesse aproveitamento considerável por parte dos estudantes, sendo estatisticamente embasados.

O fato de se trabalhar com alunos motivados em um ambiente adequado ao ensino proporcionou um espaço de efetiva troca de experiências e aprendizagem. A supervisão direta por professores e monitores facilitou a qualidade das vivências cirúrgicas possibilitando correção individualizada de técnicas erroneamente incorporadas e aquisição de novas habilidades.

Procedimentos básicos como excisão em fuso, biópsia por punch, sutura simples e correção de orelhas foram corretamente assimilados, resultando em benefício imediato ao estudante, conforme observado na tabela 2.

Alguns tipos suturas e de movimentação de tecidos careciam de conteúdos que ainda precisavam ser devidamente trabalhados durante a graduação. As técnicas que não foram completamente dominadas por este grupo podem ser justificadas pelos seguintes fatores: 1) a amostra era muito heterogênea 2 ) um quinto dos alunos situava-se entre $03^{\circ}$ e $\circ 5^{\circ}$ período, motivo pelo qual ainda não haviam cursado a disciplina de Técnica Operatória ou estivessem iniciando-a; 3) havia grande diferença entre os estudantes no manejo do material e na agilidade para execução e repetição dos exercícios; 4) a falta de uniformidade no tempo concedido aos acadêmicos e o grau de interação com os instrutores; 5) as técnicas mais complexas, como retalhos e enxertos, requeriam maior visão espacial e conhecimento anatômico prévio. As dificuldades encontradas apontaram para a necessidade de ajustes nos programas das oficinas visando seu aperfeiçoamento.

A documentação fotográfica permitiu registrar peculiaridades no manuseio do instrumental cirúrgico, especialmente pelos estudantes canhotos (Figura 2), como também, daqueles provenientes dos períodos iniciais da graduação. E serviu de base para repensar opções que contribuam para uma melhor utilização deste treinamento, possibilitando rever desde aspectos da instrumentação básica até avanços no processo de ensino-aprendizagemavaliação em cirurgia ${ }^{8}$. A filmagem da execução dos exercícios poderia, por exemplo, auxiliar na análise do gestual cirúrgico, visando corrigir erros, estimular mudanças e aprimorar o desempenho principalmente para os que pretendem seguir a ciência e arte cirúrgica.

O delineamento de pesquisa adotado permitia a intervenção do investigador na característica estudada, ou seja, no treinamento, porém não permitia a alocação aleatória dos participantes, o que constituiu uma limitação deste estudo. Isto pode ser modificado pelo redirecionamento da amostra.

Esta oficina não é uma proposta pronta e acabada, mas aberta à medida que contribui de forma inovadora como um recurso pedagógico auxiliando professores e alunos a articular a teoria com a prática. Diante das rápidas transformações tecnológicas, requer complementação contínua dos conhecimentos, habilidades e atitudes com alternativas que reproduzam inteiramente os aspectos e as condições encontradas no ato cirúrgico realizado no ser humano.

Embora restrito ${ }^{3}$, este treinamento experimental reverte em ganhos diretos para o aluno, a universidade e a população. Tem custo reduzido e respeito aos princípios éticos, é seguro e de fácil reprodução. Pode ser enriquecido e diversificado com a integração de outros modelos e ferramentas de ensino-aprendizagem em cirurgia, adaptados as necessidades especificas da graduação e até da pós-graduação.

Não existem, atualmente, trabalhos na literatura que possibilitem uma comparação com o presente estudo. Porém, os resultados obtidos nos permitem inferir que oficinas de técnica operatória cutânea, como a realizada, tem um papel fundamental no treinamento prático, e merece mais atenção na formação do graduando de medicina e na avaliação das habilidades cirúrgicas.

\title{
A $B$ S $S T$ R A C T
}

\begin{abstract}
Objective: To evaluate the effectiveness of training in surgical techniques in pig feet during medical school. Methods: We conducted a cross-sectional study with 87 volunteer medical students from different graduation semesters, who attended a basic workshop for incisions, sutures, biopsies, flaps, grafts, under direct supervision. Pre and post-training self-administered questionnaires were used. Results: The teaching of sutures, biopsies and ear correction was effective for learning of the minimal skills required for the proposed techniques ( $p<0.005)$, as well as excisions and flaps, except grafting ( $p>0$ 97). Conclusion: The methodological approach and monitoring of techniques' reproduction were adequate. The training offered complemented cutaneous surgical skills of undergraduate medicine students.
\end{abstract}

Key words: Medicine. Education. Medical education. Undergraduate medical education. Outpatient surgical procedures.

\section{REFERÊNCIAS}

1. Puentes M, Fabián F. Docencia quirúrgica: ¿como enseñas es como te ves? Arch Med. 2008;8(2):149-54.
2. Reichel JL, Peirson RP, Berg D. Teaching and evaluation of surgical skills in dermatology results of a survey. Arch Dermatol. 2004;140(11):1365-9.

3. Purim KS. Oficina de cirurgia cutânea. Rev Col Bras Cir 2010;37(4):303-5. 
4. Wang TS, Schwartz JL, Karimipour DJ, Orringer JS, Hamilton T, Jonhson TM. An education theory-based method to teach a procedural skill. Arch Dermatol. 2004;140(11):1357-61.

5. Porte MC, Xeroulis G, Reznick RK, Dubrowski A. Verbal feedback from an expert is more effective than self-accessed feedback about motion efficiency in learning new surgical skills. Am J Surg. 2007:193(1):105-10.

6. Moorthy K, Munz Y, Sarker SK, Darzi A. Objective assessment of technical skills in surgery. BMJ. 2003;327(7422):1032-7.

7. Brasil. Ministério da Educação. Resolução CNE/CES n 4, de 2001. Brasília, 2001. Diretrizes Curriculares Nacionais do Curso de Graduação em Medicina.

8. Peres CM, Andrade AS, Garcia SB. Atividades extracurriculares: multiplicidade e diferenciação necessárias ao currículo. Rev Bras educ med. 2007;31(3):203-11.
Recebido em 06/07/2012

Aceito para publicação em 10/08/2012

Conflito de interesse: nenhum

Fonte de financiamento: nenhuma.

\section{Como citar este artigo:}

Purim KSM, Santos LDS, Murara GT, Maluf EMCP, Fernandes JW, Skinovsky J. Avaliação de treinamento cirúrgico na graduação de medicina. Rev Col Bras Cir. [periódico na Internet] 2013:40(2). Disponível em URL: http://www.scielo.br/rcbc

\section{Endereço para correspondência:}

Kátia Sheylla Malta Purim

E-mail: kspurim@gmail.com 